
\title{
25 Research Soure \\ MicroRNA-200b reduced ZEB1 to inhibit cell proliferation and migration in human gastric cancer
}

\section{He Chen}

First Affiliated Hospital of Anhui Medical University

\section{Pengcheng Liu}

First Affiliated Hospital of Anhui Medical University

\section{Lei Wang}

Huaian First People's Hospital

\section{Yanxia Yu}

Anhui Medical University

Yan Zhao

First Affiliated Hospital of Anhui Medical University

\section{Yihong Cai}

Anhui Medical University

\section{Zhongxin Wang}

First Affiliated Hospital of Anhui Medical University

Min Zhang ( $\square$ ahmuzhangmin@163.com )

\section{Research article}

Keywords: microRNA-200b; proliferation; migration; gastric cancer

Posted Date: November 19th, 2019

DOI: https://doi.org/10.21203/rs.2.17514/v1

License: (c) (1) This work is licensed under a Creative Commons Attribution 4.0 International License.

Read Full License 


\section{Abstract}

BACKGROUND Gastric cancer (GC) is one of the most common malignant cancers, with high morbidity and mortality rates worldwide. The present study was to explore whether miR-200b is a tumor suppressor in GC and to unveil the potential mechanisms.METHODS Levels of c-Myc, Cyclin D1, MMP-3 and MMP-9 expression were detected respectively by q-PCR and Western blot assay. BrdU proliferation assay, Cell cycle analysis and Wound-healing were used to study the role of miR-200b with inhibitor, mimics or ZEB1RNAi in TGF-B1-treated SGC-7901/DDP cells.RESULTS Compared with the paracancerous tissues, miR200b was decreased in GC patients and SGC-7901/DDP cells. Lower level of miR-200b induced by its inhibitor promoted TGF- $\beta 1$-treated SGC-7901/DDP cells proliferation and migration, and increased the levels of c-Myc, Cyclin D1, MMP-3, and MMP-9. Interestingly, miR-200b mimics and ZEB1-RNAi were able to reduce the proliferation and migration of TGF- $\beta 1$-induced SGC-7901/DDP cells as well as their levels of c-Myc, Cyclin D1, MMP-3 and MMP-9. In addition, ZEB1 was indeed the potential target of miR-200b identified by dual luciferase reporter gene assay.CONCLUSION Taken together, our findings suggest that miR-200b be likely to play an important role in activating TGF- $\beta 1$-induced SGC-7901/DDP cells and perform as a tumor suppressor by targeting ZEB1 in GC.

\section{Background}

Gastric cancer (GC) is one of the most common malignant cancers, with high morbidity and mortality rates globally [1]. The vast majority of GC patients are often diagnosed at advanced stages since clinical symptoms of GC are not overt at early stage. The prognosis of advanced GC patients remains extremely low with the 5 -year overall survival rate of less than $30 \%$, largely due to the local and systemic metastasis of GC [2]. Therefore, it is crucial for patients with gastric cancer to discover the molecular mechanisms underlying gastric cancer development and the effective molecular biomarkers.

The microRNAs (miRNAs) are a class of endogenous and small noncoding RNAs with 18-24 nucleotides in length, which play remarkable roles in gene regulation of pathogenesis [3]. Recently, a class of miRNAs were discovered, involving important biological processes [4]. On one hand, several miRNAs apparently regulate cancer stemness of all stripes, such as miR-203 for breast cancer [5], miR-124a for non-small cell lung cancer [6], and miR-7 for prostate cancer [7]. On the other hand, accumulating evidence strongly suggests that aberrant miRNAs expression could affect the growth and metastasis of various human cancers including GC [8]. Among these miRNAs, miR-200 family members (miR-200a, miR-200b, miR200c, miR-141, and miR-429) [9], act as key inhibitors of epithelial-mesenchymal transition(EMT) by directly targeting transcriptional repressors of E-cadherin, ZEB1 and ZEB2 [10]. Interestingly, ZEB1 binds to the promoter regions of both miR-200 genomic clusters (miR-200b/200a/429 and miR-200c/141) and suppresses their transcription, suggesting that miR-200 and ZEB1 form a double-negative feedback loop to control cell migration, invasion, and EMT [11].

In this study, we investigated the differential expressions of microRNA-200b in GC and paracancerous tissues, and further explored the functions of microRNA-200b in GC. 


\section{Methods}

\section{Human gastric cancer tissue collection.}

Gastric cancer tissues $(n=6)$ in this study were obtained from patients with gastric cancer from First Affiliated Hospital, Anhui Medical University, Hefei, China. All patients signed informed consent to take part in the study. The study protocol was approved by the ethics boards of Anhui Medical University QEthics \LLSC20150030》, and tissue specimen acquisition was performed in accordance with the institutional guidelines. The written informed consent was obtained from all subjects.

\section{Reagents.}

Fetal bovine serum (FBS) was purchased from Millipore (Billerica, MA, USA). High-glucose DMEM medium was purchased from Hyclone (Logan, Utah, USA). Rabbit anti-C-Myc and rabbit anti-Cyclin D1 monoclonal antibodies were purchased from Cell Signaling (Danvers, MA, USA), Rabbit anti-MMP-9 monoclonal antibody was purchased from Millipore (Billerica, MA, USA), Rabbit anti-ZEB1 monoclonal antibody was purchased from Abcam (Eugene, USA), and anti-MMP-3 and anti- $\beta 1$-actin monoclonal antibodies were purchased from Bioworld (Shanghai, China).

\section{Cell culture}

SGC-7901 cells and SGC-7901/DDP cells were purchased from the cell bank of Chinese Academy of Sciences (Shanghai, China). The cells were cultured in cell culture flasks in DMEM (Hyclone, USA) supplemented with $10 \%(\mathrm{v} / \mathrm{v}$ ) heat-inactivated FBS (Millipore, USA), $100 \mathrm{U} / \mathrm{ml}$ of penicillin, and 100 $\mathrm{mg} / \mathrm{ml}$ of streptomycin (both from Beyotime, China). Cell cultures were maintained at $37^{\circ} \mathrm{C}$ at an atmosphere of $5 \% \mathrm{CO}_{2}$.

\section{Cells transient transfection of miR-200b and small interfering RNA silencing}

SGC-7901/DDP cells were transfected with $100 \mathrm{nM}$ of miR-200b inhibitor, miR-200b mimics (Biomics, USA), small interfering RNA (RNAi) of ZEB1 (GenePharma, Shanghai, China) using Lipofectamine 2000 (Invitrogen, CA, USA) according to the manufacturer's instructions.

\section{Cell cycle analysis}

To analyze the intracellular DNA content, SGC-7901/DDP cells were fixed in $70 \%$ ethanol at $4{ }^{\circ} \mathrm{C}$ overnight. After then, cells were stained with $0.5 \mathrm{ml}$ of propidium iodide (PI) staining buffer (Beyotime, China) containing $200 \mathrm{mg} / \mathrm{ml}$ RNase $\mathrm{A}, 50 \mu \mathrm{g} / \mathrm{ml} \mathrm{Pl}$, at room temperature for $30 \mathrm{~min}$ in dark. Flow cytometric analysis was performed on Beckman Coulter.

\section{Quantitative real-time PCR (q-PCR)}

Total RNA was extracted from cultured SGC-7901/DDP cells by using RISO RNA Isolation Reagent (Biomics, USA) according to the manufacture's protocol. Expression of miR-200b was measured using an 
EzOmics miRNA qPCR Detection Primer Set (Biomics, USA) and EzOmics One-Step qPCR Kit (Biomics, USA) in PikoReal 96 real-time PCR system (Thermo, USA). The fold-change for miR-200b relative to U6 was calculated through the 2- $\triangle \triangle C T$ method. Quantitative real-time PCR analyses of relative levels of CMyc, CyclinD1, MMP-3, MMP-9, ZEB1 and $\beta$-actin mRNA were determined using the QuantiFast SYBR Green RT-PCR kits (QIAGEN, Germany) according to the manufacturer's instruction. The sequences of primer pairs used were as follows: C-Myc (forward: 5'-GGACTATCCTGCTGCCAAGA-3'; reverse: 5'-CGCC TCTTGACATTCTCCTC-3'), CyclinD1 (forward: 5'-GATCAAGTGTGACCCGGA CT-3'; reverse: 5'TCСTССTСTTCСTССTCCTC-3'), MMP-3 (forward: 5'-GGCCA GGGATTAATGGAGAT-3'; reverse: 5'TGAAAGAGACCCAGGGAGTG-3'), MMP-9 (forward: 5'-GTACCACGGCCAACTACGAC-3'; reverse: 5'GCCTTGGA AGATGAATGGAA-3') and $\beta$-actin (forward: 5'-GCCAACACAGTGCTGTCTGG- 3'; reverse: 5'AGGAGGAGCAATGATCTTG-3').

\section{Western blot}

Cultured SGC-7901/DDP cells were lysed with lysis buffer (Beyotime, China) for Western blotting. Rabbit monoclonal antibodies directed against ZEB1 was used at 1:800, C-Myc, CyclinD1, MMP-3 and MMP-9 were used at 1:500, and $\beta$-actin was used at 1:1000. After incubated with primary antibodies, the nitrocellulose blots were washed for four times in TBS/Tween-20 before incubation in goat anti-mouse or anti-rabbit HRP-conjugating antibody at 1:10000 diluted in TBS/Tween-20 containing $5 \%$ skim milk for 1 h. After washing four times with TBS/Tween-20 the protein blots were detected using the ECLchemiluminescent kit (ECL-plus, Thermo Scientific).

\section{Luciferase reporter assays}

SGC-7901/DDP cells were cultured into 24-well plates, and transfected transiently with $200 \mathrm{ng}$ DNA of ZEB1 3'UTR WT plasmid, $60 \mathrm{nmol}$ of miR-200b mimics or the negative controls (Gene Pharma, Shanghai), using $2.25 \mu$ l of lipofectamine 2000 and $100 \mu$ l of Opti-MEM (Invitrogen, USA). The cells were harvested and lysed $48 \mathrm{~h}$ post- transfection. The luciferase activities were measured consecutively by the Dual- Luciferase Reporter 1000 Assay system (Promega, USA).

\section{Wound-healing analysis}

SGC-7901/DDP cells were cultured 2 days in 24-well plate $\left(5 \times 10^{5} / \mathrm{ml}\right.$ cells/well) in DMEM with $20 \%$ FBS. And then, the cells were serum deprived and scratched. $24 \mathrm{~h}$ later Type II alveolar epithelial cells were fixed with methanol, stained with crystal violet stain, and viewed under an Olympus BX-51 microscope.

\section{Statistical analysis.}

Data were presented as means \pm SD and analyzed using SPSS16.0 software. Statistical significances were determined by one-way ANOVA with the post-hoc Dunnett's test. In all assays, values of $P<0.05$ were considered to be statistically significant. 


\section{Results}

1. The expression of miR-200b was down-regulated in GC and TGF- 31 -induced SGC-7901/DDP cells.

To ascertain the role of miR-200b in gastric carcinoma, gastric cancer tissues were collected. As shown in Fig. $1 \mathrm{~A}$, the level of miR-200b was decreased in GC compared with the paracancerous tissues by qRTPCR. Likewise, compared with SGC-7901, the miR-200b level was also reduced in SGC-7901/DDP cells. Moreover, the expression of miR-200b was down-regulated by various concentrations of TGF- $\beta 1$ in SGC7901/DDP cells (Fig. 1 B). These results demonstrated that the miR-200b may be closely associated with the incidence of gastric cancer.

2. The miR-200b inhibitor enhanced proliferation and migration of TGF- $\beta 1$-induced SGC-7901/DDP cells

To further identify the functions of miR-200b, we assessed the effect of miR-200b on the proliferation and migration of TGF- $\beta 1$-induced SGC-7901/DDP cell lines through miR-200b inhibitor. Firstly, downregulation of miR-200b in TGF- $\beta 1$-induced SGC-7901/DDP cells was verified using qRT-PCR (Fig. 1C). In addition, c-Myc and Cyclin D1 oncogenes were detected to increase (Figs. 1D and 1E) by miR-200b inhibitor. Notably, BrdU cell proliferation ELISA assay showed that the TGF- $\beta 1$-induced SGC-7901/DDP cells were promoted to proliferate considerably with miR-200b inhibitor (Fig. 1G). Moreover, as shown in Fig. $1 \mathrm{~F}$, we found that the ratios of cells in $\mathrm{G} 2 / \mathrm{M}$ phases were increased after transfect with miR-200b inhibitor. More significantly, MMP-3 and MMP-9 mRNA and protein expression were observed to upregulate by miR-200b inhibitor in TGF- $\beta 1$-induced SGC-7901/DDP cells (Figs. 1H and 1I). Remarkably, wound-healing assay proved that the TGF- $\beta 1$-induced SGC-7901/DDP cell migration was augmented via miR-200b inhibitor (Fig. 1J). Thus, these data indicate that miR-200b inhibitor be potential to dramatically enhance the ability of TGF- $\beta 1$-induced SGC-7901/DDP cell proliferation and migration.

3. The miR-200b mimics weakened proliferation and migration of TGF- $\beta 1$-induced SGC-7901/DDP cells

In order to provide additional evidence that miR-200b involves in the proliferation and migration of TGF$\beta 1$-induced SGC-7901/DDP cells, miR-200b mimics were used to over-express miR-200b. $48 \mathrm{~h}$ after treating TGF- $\beta 1$-induced SGC-7901/DDP cells with miR-200b mimics, the miR-200b mRNA level was increased remarkably compared with the control (Fig. 2H). Meanwhile, c-Myc and cyclin D1 expressions were decreased by miR-200b mimics in TGF- $\beta 1$-induced SGC-7901/DDP cells (Figs. 2A and 2B). BrdU cell proliferation ELISA assay showed that the proliferation of cells was inhibited observably by miR-200b mimics (Fig. 2D). In addition, flow cytometric analysis showed over-expression of miR-200b resulted in the pronounced decrease of the proportions of cells in G2/M phase (Fig. 2C). On the other hand, the expressions of MMP-3 and MMP-9 were down-regulated significantly by miR-200b mimics in TGF- $\beta 1$ induced SGC-7901/DDP cells as shown in Figs. 2E and 2F. To be noted, wound-healing assay proved that the cell migration was inhibited considerably with miR-200b mimics (Fig. 2G). These results indicate that 
miR-200b mimics weaken the proliferation and migration of TGF- $\beta 1$-induced SGC-7901/DDP cells. The miR-200b might be related to the SGC-7901/DDP cell proliferation and migration, playing a vital role in the pathogenesis of GC.

4. ZEB1 was a target gene of miR-200b.

To next explore the underlying mechanisms of miR-200b in SGC-7901/DDP cells, bioinformatics analysis (miRanda, miRbase Targets and TargetScan) was applied to predicting miR-200b possible targets. These three approaches predicted that miR-200b was complementary to 3' non-coding regions of ZEB1 mRNA. In addition, it was found that the levels of ZEB1 protein and mRNA were both high in gastric carcinoma samples (Figs. 3A and 3B). Compared with SGC-7901 cells, the expression of ZEB1 was also up-regulated in SGC-7901/DDP cells as shown in Fig. 3C and 3E. Moreover, it was also increased significantly by TGF$\beta 1$ in SGC-7901/DDP cells (Figs. 3C - 3E). Moreover, the level of ZEB1 was increased significantly by the miR-200b inhibitor (Figs. $3 \mathrm{G}$ and $3 \mathrm{H}$ ), and decreased substantially by the miR-200b mimics in TGF- $\beta 1$ induced SGC-7901/DDP cells (Figs. $3 I$ and 3J). To confirm the effects of miR-200b on ZEB1 mRNA, ZEB1WT 3'UTR was constructed and co-transfected with miR-200b mimics into SGC-7901/DDP cell, which caused the changes of fluorescence signals. As compared to the control, the co-transfection with miR200b mimics significantly suppressed the ZEB1 3'UTR activity (Fig. 3F). In conclusion, miR-200b suppressed the expression of ZEB1 by specifically binding to ZEB1 mRNA.

5. Down-regulation of ZEB1 weakened proliferation and migration of TGF- 31 -induced SGC-7901/DDP cells

To definitely determine how ZEB1 influences the proliferation and migration of SGC-7901/DDP cells, human ZEB1 specific siRNA was obtained to knockdown the ZEB1 expression in SGC-7901/DDP cells (Figs. 4A, C and D). The results showed that C-Myc and CyclinD1 levels were remarkably reduced in TGF$\beta 1$-induced SGC-7901/DDP cells treated with the ZEB1 RNAi (Figs. 4C and 4D). Similar to the miR-200b mimics, the ZEB1-RNAi significantly reduced the proportions of G2/M phases in TGF- $\beta 1$-induced SGC7901/DDP cells as shown in Fig. 4E. In addition, BrdU cell proliferation ELISA showed that the cell proliferation was inhibited substantially by the ZEB1-RNAi (Fig. 4B). Meanwhile, MMP-3 and MMP-9 (Figs. 4F and 4G) were also decreased by the ZEB1-RNAi in TGF- 31 -induced SGC-7901/DDP cells. Moreover, wound-healing assay exhibited that the cell migration ability was inhibited significantly with the ZEB1-RNAi (Fig. 4H). All the findings suggest that ZEB1-RNAi be likely to weaken the ability of TGF$\beta 1$-induced SGC-7901/DDP cells proliferation and migration.

6. miR-200b may modulate proliferation and migration of by SGC-7901/DDP Wnt/ $\beta$-catenin signaling pathway 
We also investigated the effect of miR-200b on Wnt/ $\beta$-catenin signaling in SGC-7901/DDP cells. Western blot have performed that the expression of $\beta$-catenin was up-regulated obviously by miR-200b inhibitor, and down-regulated obviously by miR-200b mimics in TGF- $\beta 1$-induced SGC-7901/DDP cells, as show as Fig.5A and B. In particular, Fig.5C showed that ZEB1-RNAi also reduced the expreesion of $\beta$-catenin. Taken together, all the above results indicated that miR-200b could modulate proliferation and migration of TGF- $\beta 1$-induced SGC-7901/DDP cells by activation of Wnt/ $\beta$-catenin signaling pathway.

\section{Discussion}

Many researchers have proved that miRNAs are involved in many diseases and biological processes through inhibiting target genes expression, including cell proliferation, differentiation and apoptosis [4]. Abnormal expression of certain microRNAs were observed in some cancers compared with normal tissues, such as endometrial carcinoma [12], breast cancer [13], ovarian cancer [14], gastric cancer [15], hepatocellular carcinoma [16] et al. Various miRNAs may act as oncogenes or tumor suppressor genes respectively according to their roles in tumorigenesis [17]. For oncogenic miRNA, the inhibitor of miRNA blocks its functions; while tumor suppressive miRNA including miRNA precursor produces anti-tumor effects. It has been proved that many miRNAs came into play in GC including miR-15b, miR-16 [18], miR21 [19], miR-23a [20], miR-146a [21], miR-200 family [22] et al. MiR-200b is widely expressed in many human malignant tumors, including endometrial carcinoma, breast cancer, colorectal cancer, pancreatic cancer, and GC [23]. It indicates that miR-200b be playing a dual role of oncogene or tumor suppressor by regulating target genes, which provides a new alternative for treatment in the course of different tumorigenesis and development. Interestingly, miRNA-200b could inhibit the proliferation, invasion, and migration of glioma cells possibly through targeting CD133 in glioma [24]. Moreover, Chen [25] has proved DTIC inhibited the growth of melanoma cells by up-regulating expression of miRNA-200.

The previous study showed that the levels of miRNA-200b were decreased in GC tissues compared with paracancerous tissues by qRT-PCR. Wu et al. [26] also found miRNA-200 family (miRNA-200a, miRNA$200 \mathrm{~b}$ and miRNA-200c) was down-regulated in GC. Moreover, the miR-200b was also down-regulated in SGC-7901/DDP compared with SGC-7901 cells. Hence, we supposed that miR-200b would play a pivotal role in GC. We also proved that the expression of miR-200b was decreased by various concentrations of TGF- $\beta 1$ in SGC-7901/DDP cells. Indeed, it is necessary to consider that miR-200b is likely to be closely related to the tumorigenesis of GC.

To identify the effects of miR-200b in GC, miR-200b inhibitor was used to lessen miR-200b expression in TGF- $\beta 1$-induced SGC-7901/DDP cells. It is generally known that C-Myc and Cyclin D1 are important downstream signaling molecules in cell proliferation pathway. C-Myc plays an important part in regulating G-phase of cell cycle as proto-oncogene protein, while Cyclin D, acting on G1-phase, can promote G1/S phase transition and accelerate the process of cell cycle [27]. We chose c-Myc and Cyclin D1 as indicators of proliferation of SGC-7901/DDP cells. The results illustrated that low-expression of miR-200b with miR-200b inhibitor up-regulated c-Myc and Cyclin D1 in TGF- $\beta 1$-induced SGC-7901/DDP cells. Moreover, BrdU cell proliferation assay also showed that the miR-200b inhibitor was able to 
promote proliferation of TGF- $\beta 1$-induced SGC-7901/DDP cells. And cell cycle analysis showed that miR$200 \mathrm{~b}$ inhibitor led to the increased proportions of $\mathrm{G} 2 / \mathrm{M}$ phase, which was consistent with the results of cell proliferation. Migration is another important biological process in malignant tumors. Multiple studies have confirmed that cell migration was related to matrix metalloproteinases (MMPs) $[28,29]$. More significantly, MMP-3 and MMP-9 mRNA and protein levels were up-regulated significantly after transfection with miR-200b inhibitor. Remarkably, wound-healing assay further proved that the cell migration was dramatically promoted with miR-200b inhibitor. These data suggest that down-regulation of miR-200b might enhance both proliferation and migration abilities of TGF- $\beta 1$-induced SGC-7901/DDP cells. The uncontrollable proliferation of cells is always corresponding with maladjusted cell cycle and shorter passage time [30].

Next, we designed gain-of-function study by transfecting miR-200b mimics into TGF- $\beta 1$-induced SGC7901/DDP cells to simulate endogenous microRNAs and it up-regulated the level of miR-200b. The results showed that up-regulation of miR-200b decreased c-Myc, Cyclin D1, MMP-3 and MMP-9 expressions in TGF- $\beta 1$-induced SGC-7901/DDP cells. Similarly, the percentages of TGF- $\beta 1$-induced SGC$7901 /$ DDP cells at G2/M stages were detected to decrease by FACS. Moreover, BrdU cell proliferation assay revealed that the cell proliferation was inhibited considerably by miR-200b mimics. Wound-healing assay proved that the cell migration ability was significantly weakened with miR-200b mimics. These findings indicate that over-expression of miR-200b could significantly inhibit the proliferation and migration of TGF- $\beta 1$-induced SGC-7901/DDP cells.

In this study, bioinformatics analysis exhibited that ZEB1 mRNA 3'UTR was complementary with the miR200b. Through the construction and transfection of ZEB1 3'UTR-WT and miR-200b mimics into SGC7901/DDP cells, which caused the fluorescence changes, ZEB1 was eventually identified as the potential target of miR-200b. Title et al. [11] reported that disrupting the in vivo regulation of ZEB1 by miR-200 was sufficient to drive EMT in tumor progression and invasion. ZEB1 is a critical member of the ZEB family of transcription factors [31], involved in a regulation of key factors during malignant cells at the invasive front of carcinomas by triggering EMT. EMT could control protein stabilization mechanisms, transcription and translation, alternative splicing and expression of non-coding RNAs [32]. In the present study, after treating the SGC-7901/DDP cells with the miR-200 inhibitor, the mRNA and protein expressions of ZEB1 were up-regulated in GC. Similarly, SGC-7901/DDP cells transfected with miR-200 mimics significantly inhibited the ZEB1 mRNA and protein levels compared with the control. Coincidentally, we found the ZEB1 mRNA and protein levels were over-expressed in GC samples. Moreover, it was also increased significantly by TGF- $\beta 1$ in SGC-7901/DDP cells. Additionally, inhibition of ZEB1 induced by ZEB1-RNAi significantly suppressed the mRNA and protein expressions of c-Myc, CyclinD1, MMP-3 and MMP-9 in TGF- $\beta 1$-induced SGC-7901/DDP cells. Interestingly, ZEB1-RNAi reduced the proportion of G2/M-phase cells remarkably. Notably, BrdU cell proliferation assay presented that the cell proliferation was inhibited significantly by the ZEB1-RNAi. Wound-healing assay further proved that the cell migration was hindered dramatically with the ZEB1-RNAi. These results indicate that the proliferation and migration abilities were compromised in TGF- $\beta 1$-induced SGC-7901/DDP cells after transfection with the ZEB1-RNAi. Zhou et al. [33] also found that miR-200c/141 decreased ZEB1/2 and increased E-cadherin expressions to repress 
the migration and invasion of gastric cancer cells. Therefore, we speculate that miR-200b is likely to inhibit cell proliferation and migration of TGF- $\beta 1$-induced SGC-7901/DDP cells through targeting ZEB1.

\section{Conclusions}

In conclusion, as our study demonstrated, consistent with experiments in vitro, miR-200b plays an important role in activating TGF- 31 -induced SGC-7901/DDP cells and may perform as a tumor suppressor by targeting ZEB1 in GC. Therefore, it is likely to provide new insight for the diagnosis and new targeted therapy of GC.

\section{Abbreviations}

EMT: epithelial-mesenchymal transition; FBS: Fetal bovine serum; GC: Gastric cancer; miRNAs: microRNAs; MMPs: matrix metalloproteinases; PI: propidium iodide; q-PCR: quantitative real-time PCR.

\section{Declarations}

\section{Ethics approval and consent to participate}

This study was approved by ethical committee of Anhui Medicine University.

\section{Consent for publication.}

Not applicable.

\section{Availability of data and material}

The data sets used and/or analyzed during the current study available from the corresponding author on reasonable request.

\section{Competing interests}

The authors declare that they have no competing interests.

\section{Funding}


This work was supported by National Natural Science Foundation of China (Grants No. 81572801) and Natural Science Foundation of Anhui Province (Grants No. KJ2017A208).

\section{Authors' contributions}

HC, ZXW, YHC and MZ conceived and designed the study. HC, PCL, LW, YXY and YZ performed the experiments. $\mathrm{HC}$ and PCL analyzed the data. $\mathrm{HC}, \mathrm{MZ}, \mathrm{ZXW}$ and $\mathrm{YZ}$ drafted the manuscript. All authors read and approved the final manuscript.

\section{Acknowledgements}

Our thanks are given to Dr. Jinxing Xia for his valuable comments to the manuscript.

\section{References}

1. Liu Y, Fan D: Ginsenoside Rg5 induces $\mathbf{G} 2 / \mathrm{M}$ phase arrest, apoptosis and autophagy via regulating ROS-mediated MAPK pathways against human gastric cancer. Biochem Pharmacol 2019, 168:285304.

2. Wu WW, Zhang WY, Zhang WH, Yang L, Deng XQ, Ou MC, Yang YX, Liu HB, Zhu T: Survival analysis of intraoperative blood salvage for patients with malignancy disease: A PRISMA-compliant systematic review and meta-analysis. Medicine (Baltimore) 2019, 98(27):e16040.

3. Spizzo R, Nicoloso MS, Croce CM, Calin GA: SnapShot: MicroRNAs in Cancer. Cell 2009, 137(3):586586 e581.

4. Budak H, Akpinar BA: Plant miRNAs: biogenesis, organization and origins. Funct Integr Genomics 2015, 15(5):523-531.

5. Muhammad N, Bhattacharya S, Steele R, Ray RB: Anti-miR-203 suppresses ER-positive breast cancer growth and stemness by targeting SOCS3. Oncotarget 2016, 7(36):58595-58605.

6. Yu F, Liu JB, Wu ZJ, Xie WT, Zhong XJ, Hou LK, Wu W, Lu HM, Jiang XH, Jiang JJ et al: Tumor suppressive microRNA-124a inhibits stemness and enhances gefitinib sensitivity of non-small cell lung cancer cells by targeting ubiquitin-specific protease 14. Cancer Lett 2018, 427:74-84.

7. Paccez JD, Duncan K, Sekar D, Correa RG, Wang Y, Gu X, Bashin M, Chibale K, Libermann TA, Zerbini LF: Dihydroartemisinin inhibits prostate cancer via JARID2/miR-7/miR-34a-dependent downregulation of Axl. Oncogenesis 2019, 8(3):14.

8. Li M, Gao M, Xie X, Zhang Y, Ning J, Liu P, Gu K: MicroRNA-200c reverses drug resistance of human gastric cancer cells by targeting regulation of the NER-ERCC3/4 pathway. Oncol Lett 2019, 18(1):145152. 
9. Peng L, Fu J, Ming Y: The miR-200 family: multiple effects on gliomas. Cancer Manag Res 2018, 10:1987-1992.

10. Jauhari A, Yadav S: MiR-34 and MiR-200: Regulator of Cell Fate Plasticity and Neural Development. Neuromolecular Med 2019, 21(2):97-109.

11. Title AC, Hong SJ, Pires ND, Hasenohrl L, Godbersen S, Stokar-Regenscheit N, Bartel DP, Stoffel M: Genetic dissection of the miR-200-Zeb1 axis reveals its importance in tumor differentiation and invasion. Nat Commun 2018, 9(1):4671.

12. Bao W, Wang HH, Tian FJ, He XY, Qiu MT, Wang JY, Zhang HJ, Wang LH, Wan XP: A TrkB-STAT3-miR204-5p regulatory circuitry controls proliferation and invasion of endometrial carcinoma cells. $\mathrm{Mol}$ Cancer 2013, 12:155.

13. Rhodes LV, Martin EC, Segar HC, Miller DF, Buechlein A, Rusch DB, Nephew KP, Burow ME, CollinsBurow BM: Dual regulation by microRNA-200b-3p and microRNA-200b-5p in the inhibition of epithelial-to-mesenchymal transition in triple-negative breast cancer. Oncotarget 2015, 6(18):1663816652.

14. Vilming Elgaaen B, Olstad OK, Haug KB, Brusletto B, Sandvik L, Staff AC, Gautvik KM, Davidson B: Global miRNA expression analysis of serous and clear cell ovarian carcinomas identifies differentially expressed miRNAs including miR-200c-3p as a prognostic marker. BMC Cancer 2014, 14:80.

15. Li HL, Xie SP, Yang YL, Cheng YX, Zhang Y, Wang J, Wang Y, Liu DL, Chen ZF, Zhou YN et al: Clinical significance of upregulation of mir-196a-5p in gastric cancer and enriched KEGG pathway analysis of target genes. Asian Pac J Cancer Prev 2015, 16(5):1781-1787.

16. Yao S, Tian C, Ding Y, Ye Q, Gao Y, Yang N, Li Q: Down-regulation of Kruppel-like factor-4 by microRNA-135a-5p promotes proliferation and metastasis in hepatocellular carcinoma by transforming growth factor-beta1. Oncotarget 2016, 7(27):42566-42578.

17. Boufraqech M, Klubo-Gwiezdzinska J, Kebebew E: MicroRNAs in the thyroid. Best Pract Res Clin Endocrinol Metab 2016, 30(5):603-619.

18. Cimmino A, Calin GA, Fabbri M, lorio MV, Ferracin M, Shimizu M, Wojcik SE, Aqeilan RI, Zupo S, Dono $M$ et al: miR-15 and miR-16 induce apoptosis by targeting BCL2. Proc Natl Acad Sci U S A 2005, 102(39):13944-13949.

19. Zhang BG, Li JF, Yu BQ, Zhu ZG, Liu BY, Yan M: microRNA-21 promotes tumor proliferation and invasion in gastric cancer by targeting PTEN. Oncol Rep 2012, 27(4):1019-1026.

20. Liu X, Ru J, Zhang J, Zhu LH, Liu M, Li X, Tang H: miR-23a targets interferon regulatory factor 1 and modulates cellular proliferation and paclitaxel-induced apoptosis in gastric adenocarcinoma cells. PLoS One 2013, 8(6):e64707.

21. Kogo R, Mimori K, Tanaka F, Komune S, Mori M: Clinical significance of miR-146a in gastric cancer cases. Clin Cancer Res 2011, 17(13):4277-4284.

22. Shinozaki A, Sakatani T, Ushiku T, Hino R, Isogai M, Ishikawa S, Uozaki H, Takada K, Fukayama M: Downregulation of microRNA-200 in EBV-associated gastric carcinoma. Cancer Res 2010, 
70(11):4719-4727.

23. Feng B, Wang R, Chen LB: Review of miR-200b and cancer chemosensitivity. Biomed Pharmacother 2012, 66(6):397-402.

24. Zhao C, Ma ZG, Mou SL, Yang YX, Zhang YH, Yao WC: Targeting effect of microRNA on CD133 and its impact analysis on proliferation and invasion of glioma cells. Genet Mol Res 2017, 16(1).

25. Chen YN: Dacarbazine inhibits proliferation of melanoma FEMX-1 cells by up-regulating expression of miRNA-200. Eur Rev Med Pharmacol Sci 2017, 21(6):1191-1197.

26. Wu XM, Shao XQ, Meng XX, Zhang XN, Zhu L, Liu SX, Lin J, Xiao HS: Genome-wide analysis of microRNA and mRNA expression signatures in hydroxycamptothecin-resistant gastric cancer cells. Acta Pharmacol Sin 2011, 32(2):259-269.

27. Zheng L, Liang X, Li S, Li T, Shang W, Ma L, Jia X, Shao W, Sun P, Chen C et al: CHAF1A interacts with TCF4 to promote gastric carcinogenesis via upregulation of C-MYC and CCND1 expression. EBioMedicine 2018, 38:69-78.

28. Wells JM, Gaggar A, Blalock JE: MMP generated matrikines. Matrix Bio/ 2015, 44-46:122-129.

29. Tan C, Qiao F, Wei P, Chi Y, Wang W, Ni S, Wang Q, Chen T, Sheng W, Du X et al: DIXDC1 activates the Wnt signaling pathway and promotes gastric cancer cell invasion and metastasis. Mol Carcinog 2016, 55(4):397-408.

30. Scatena R: Mitochondria and cancer: a growing role in apoptosis, cancer cell metabolism and dedifferentiation. Adv Exp Med Biol 2012, 942:287-308.

31. Zhang Y, Xu L, Li A, Han X: The roles of ZEB1 in tumorigenic progression and epigenetic modifications. Biomed Pharmacother 2019, 110:400-408.

32. Singh M, Yelle N, Venugopal C, Singh SK: EMT: Mechanisms and therapeutic implications. Pharmacol Ther 2018, 182:80-94.

33. Zhou X, Wang Y, Shan B, Han J, Zhu H, Lv Y, Fan X, Sang M, Liu XD, Liu W: The downregulation of miR-200c/141 promotes ZEB1/2 expression and gastric cancer progression. Med Onco/ 2015, 32(1):428.

\section{Figures}


A
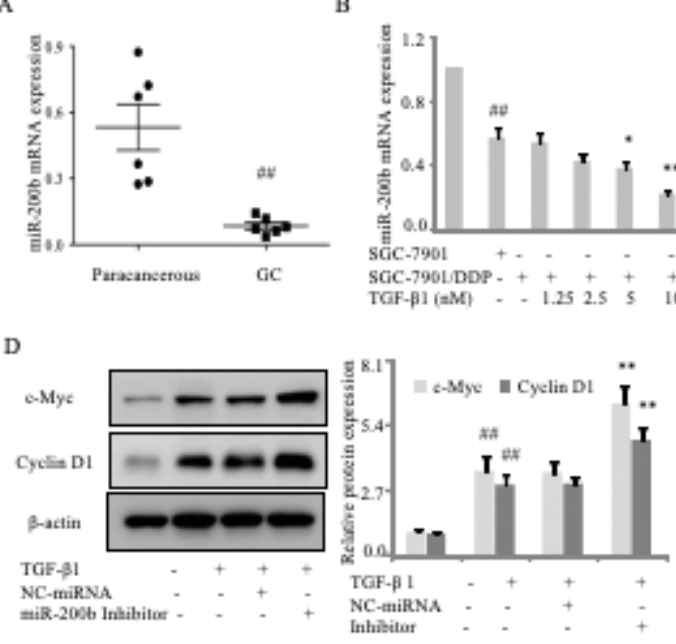

F

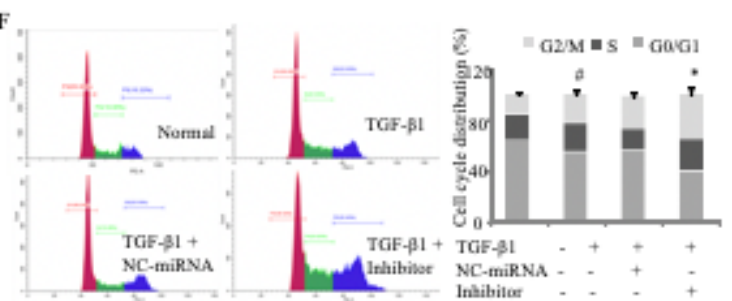

H

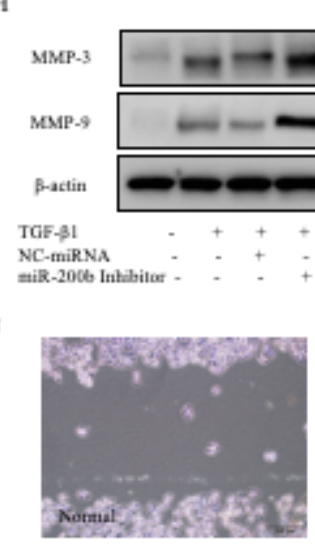

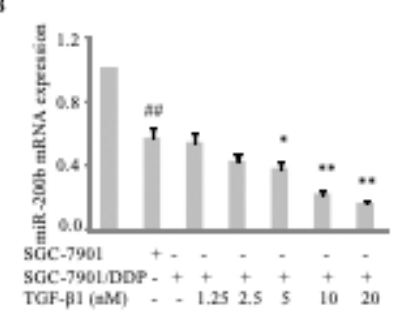
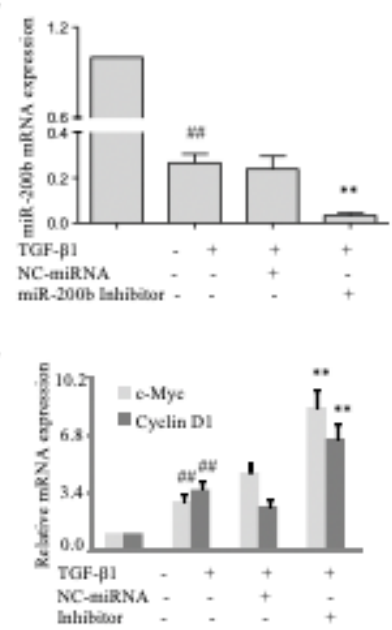

G

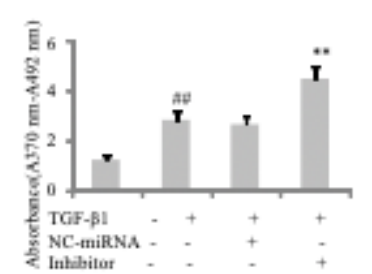

1

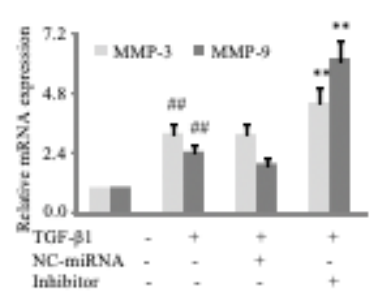

\section{Figure 1}

miR-200b inhibitor increased proliferation and migration of TGF- $\beta 1$-induced SGC-7901/DDP cells (A) and (B) The mRNA level of miR-200b was determined. (C) The mRNA expression level of miR-200b in TGF- $\beta 1$ induced SGC-7901/DDP cells with miR-200b inhibitor. (D) and (E) The levels of c-Myc and Cyclin D1. (F) Cell cycle of SGC-7901/DDP cells. (G) BrdU proliferation assay. $(\mathrm{H})$ and $(\mathrm{I})$ The expression levels of MMP3 and MMP-9. (J) Cell migration in SGC-7901/DDP cells (original magnification, 10X). All the data were expressed in the form of means \pm SD. \#P $<0.05$, \#\#P $<0.01$ vs. paracancerous, SGC-7901 or normal group; ${ }^{*}<0.05,{ }^{\star *} \mathrm{P}<0.01$ vs. SGC-7901/DDP or NC-miRNA group. 
A

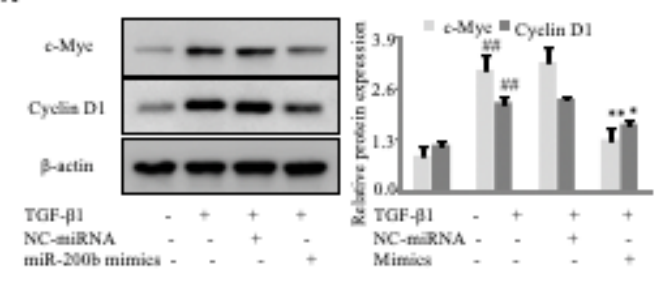

c

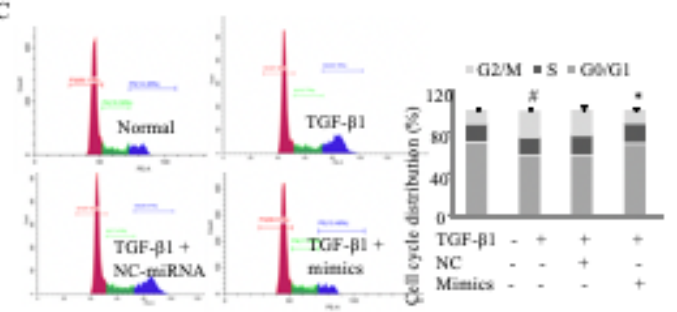

E

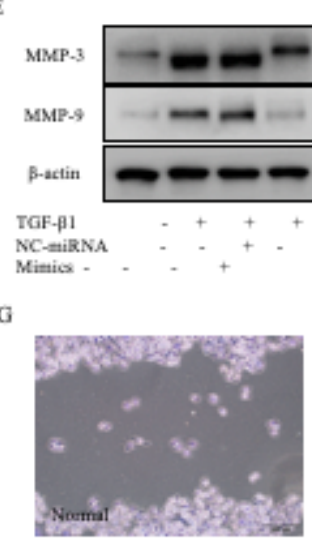

H
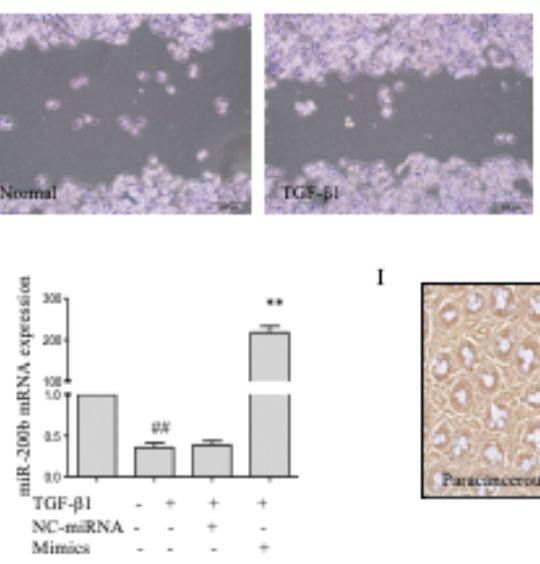

B
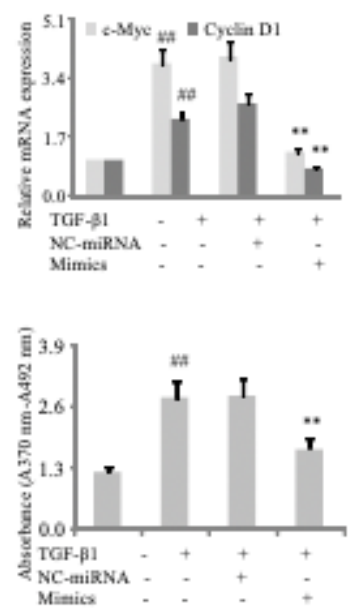

$\mathrm{F}$

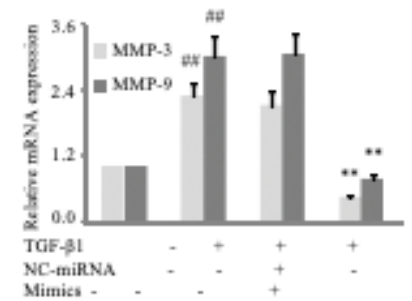

Mimist-
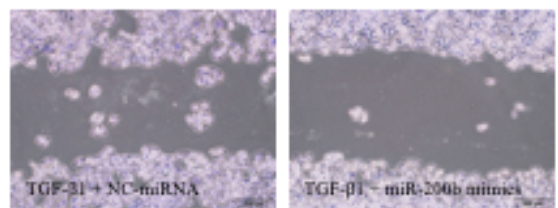

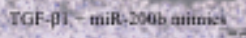

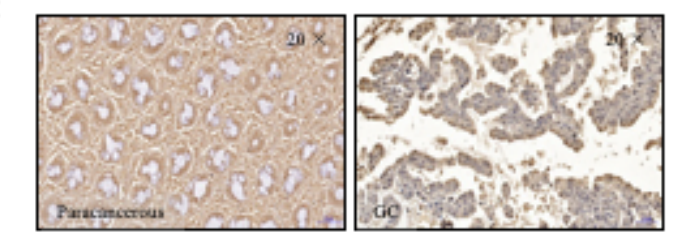

Figure 2

miR-200b mimics inhibited proliferation and migration of TGF- 31 -induced SGC-7901/DDP cells. (A) and (B) The levels of C-Myc and Cyclin D1. (C) Cell cycle. (D) BrdU proliferation assay. (E) and (F) The levels of MMP-3 and MMP-9. (G) Cell migration (original magnification, 10X). (H) The mRNA expression level of miR-200b. (I) The expressions of ZEB1 in paracancerous and GC tissues were analyzed by IHC staining analysis (original magnification, 20X). \#P<0.05, \#\#P<0.01 vs. normal group; ${ }^{*}<<0.05,{ }^{\star *} \mathrm{P}<0.01 \mathrm{vs}$. NC-miRNA. 


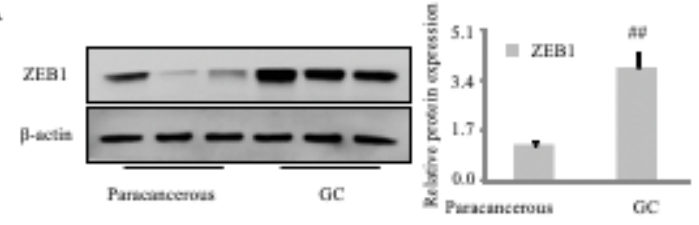

$\mathrm{C}$

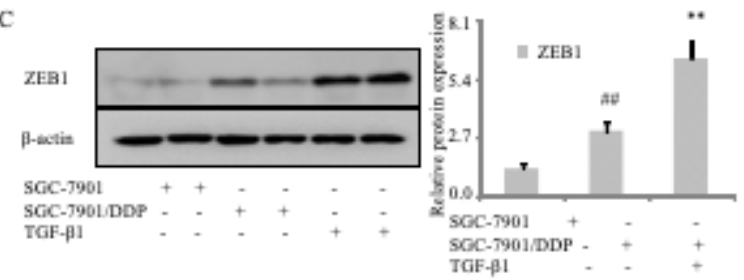

E

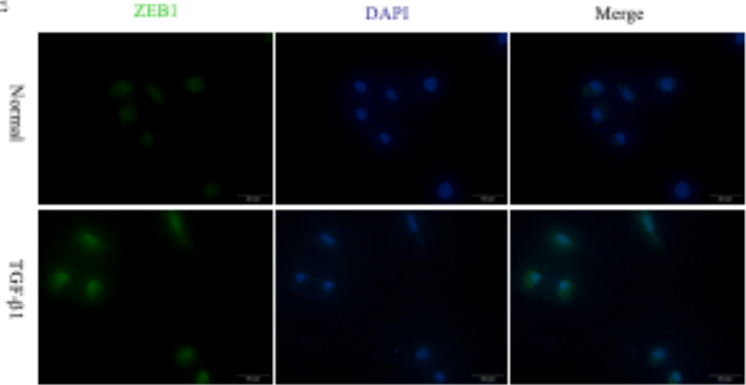

G
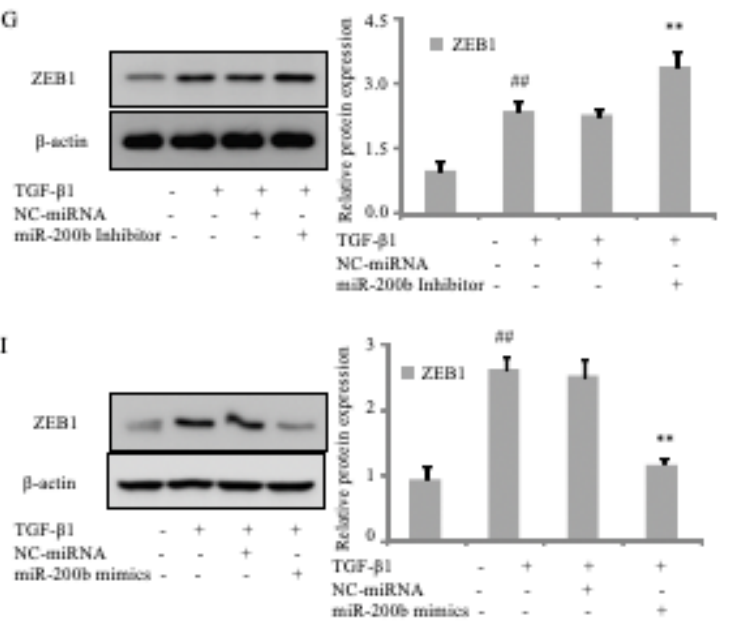

B

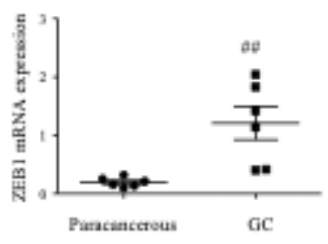

D
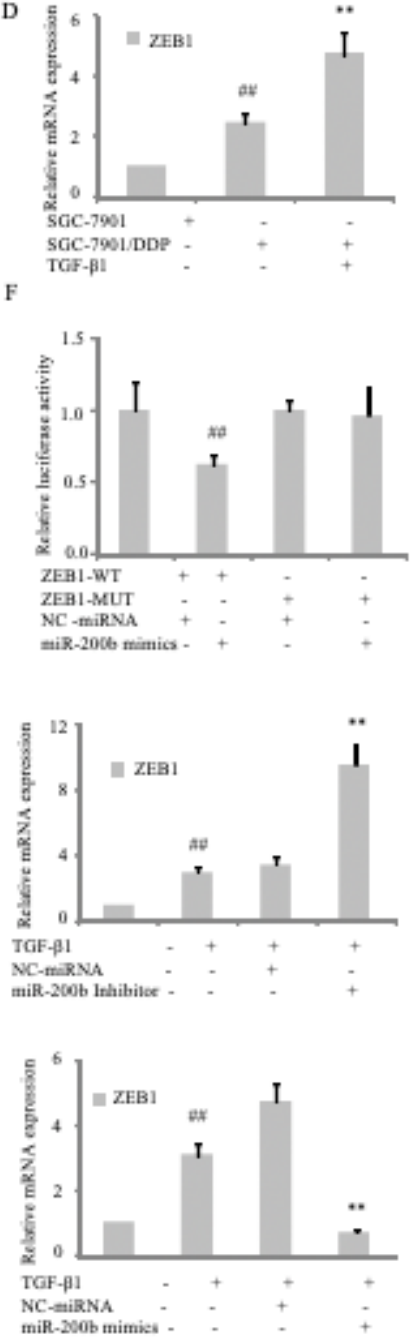

\section{Figure 3}

ZEB1 was a direct target of miR-200b. (A) - (D) The expression of ZEB1. (E) The protein expression of ZEB1 was detected by immunofluorescence analysis in SGC-7901/DDP cells treated with TGF- $\beta 1$. (F) ZEB1 3' UTR and miR-200b mimics in SGC-7901/DDP cells were tested by Dual luciferase assay. (G) - (J) The expression of ZEB1. \#\#P $<0.01$ vs. paracancerous, SGC-7901 or NC group; **P < 0.01 vs. SGC7901/DDP or NC-miRNA group. 
A
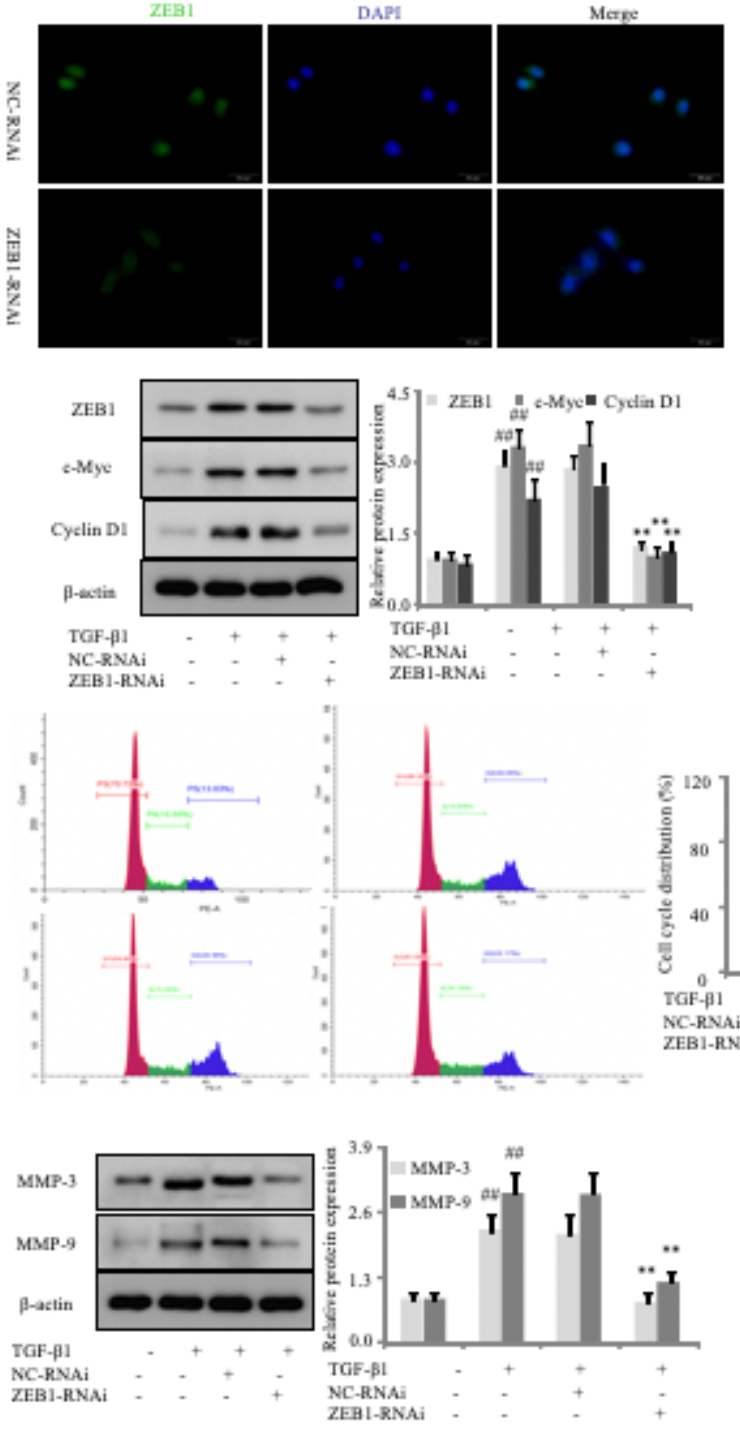

E

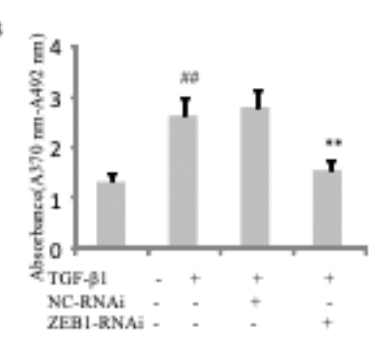

D

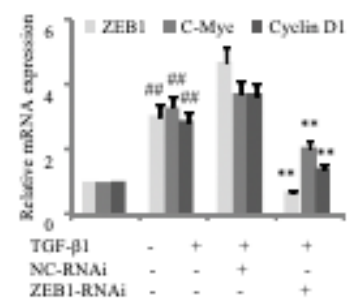

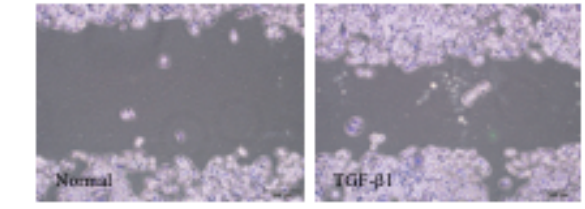
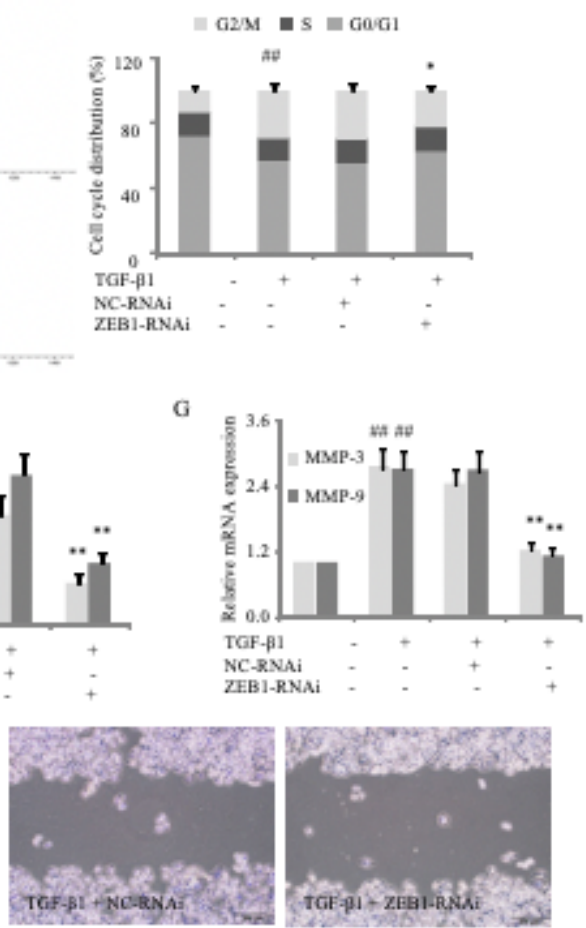

Figure 4

ZEB1-RNAi inhibited proliferation and migration of TGF- 31 -induced SGC-7901/DDP cells. (A) The expression of ZEB1 was detected by immunoflurescence. (B) BrdU proliferation assay. (C) and (D) The levels of ZEB1, c-Myc and Cyclin D1. (E) Cells cycle (F) and (G) The levels of MMP-3 and MMP-9. (H) Cells migration (original magnification, $10 \mathrm{X}$ ). \#\#P $<0.01$ vs. normal group; ${ }^{\star \star} \mathrm{P}<0.01$ vs. NC-RNAi. 
A

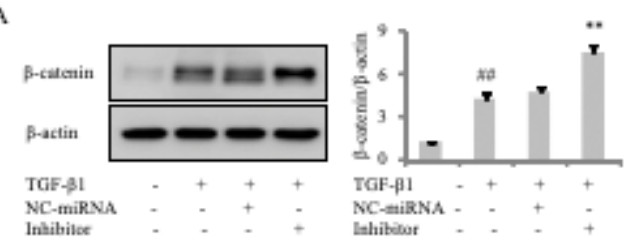

B
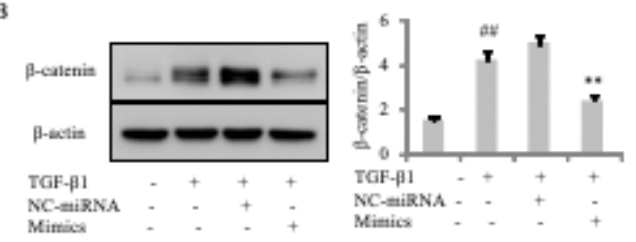

C

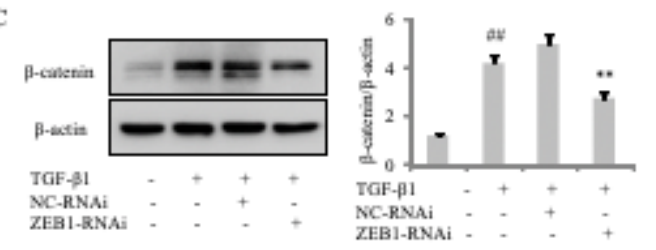

\section{Figure 5}

miR-200b may modulate Wnt/ $\beta$-catenin signaling pathway in TGF- $\beta 1$-induced SGC-7901/DDP cells. (A) The expression of $\beta$-catenin in TGF- $\beta 1$-induced SGC-7901/DDP cells with miR-200b inhibitor. (B) The expression of $\beta$-catenin in TGF- $\beta 1$-induced SGC-7901/DDP cells with miR-200b mimics. (C) The expression of $\beta$-catenin in TGF- $\beta 1$-induced SGC-7901/DDP cells with ZEB1-RNAi. \#\#P< 0.01 vs. normal group; $* * P<0.01$ vs. NC-RNAi. 